The polymyxin derivative NAB739 is synergistic with several antibiotics against polymyxin-resistant strains of Escherichia coli, Klebsiella pneumoniae and Acinetobacter baumannii

Tyrrell, Jonathan M.

2019-02

Tyrrell , J M , Aboklaish , A F , Walsh , T R , Vaara , T \& Vaara , M 2019 , ' The polymyxin derivative NAB739 is synergistic with several antibiotics against polymyxin-resistant strains of Escherichia coli, Klebsiella pneumoniae and Acinetobacter baumannii ' , Peptides, vol. 112 , pp. 149-153 . https://doi.org/10.1016/j.peptides.2018.12.006

http://hdl.handle.net/10138/313768

https://doi.org/10.1016/j.peptides.2018.12.006

publishedVersion

Downloaded from Helda, University of Helsinki institutional repository.

This is an electronic reprint of the original article.

This reprint may differ from the original in pagination and typographic detail.

Please cite the original version. 


\title{
The polymyxin derivative NAB739 is synergistic with several antibiotics against polymyxin-resistant strains of Escherichia coli, Klebsiella pneumoniae and Acinetobacter baumannii
}

\author{
Jonathan M. Tyrrell ${ }^{\mathrm{a}}$, Ali F. Aboklaish ${ }^{\mathrm{a}}$, Timothy R. Walsh ${ }^{\mathrm{a}}$, Timo Vaara ${ }^{\mathrm{b}}$, Martti Vaara ${ }^{\mathrm{b}, \mathrm{c}, *}$ \\ ${ }^{a}$ Department of Medical Microbiology and Infectious Disease, Cardiff University Medical School, Cardiff, Wales, United Kingdom \\ ${ }^{\mathrm{b}}$ Northern Antibiotics Ltd, FI-02150, Espoo, Finland \\ ${ }^{\mathrm{c}}$ Department of Bacteriology and Immunology, Helsinki University Medical School, FI-00014, Helsinki, Finland
}

A R T I C L E I N F O

\section{Keywords:}

Polymyxin B

NAB739

Klebsiella pneumoniae

Escherichia coli

Acinetobacter baumannii

mcr-1

Polymyxin resistance

Rifampin

Retapamulin

Meropenem

\begin{abstract}
A B S T R A C T
The antibiotic crisis has reinstated polymyxins, once abandoned because of their toxicity. Now, preclinical studies have revealed better tolerated and more effective derivatives of polymyxins such as NAB739. Simultaneously, polymyxin-resistant (PMR) strains such as the $m c r-1$ strains have received lots of justified publicity, even though they are still very rare. Here we show that NAB739 sensitizes the PMR strains to rifampin, a classic "anti-Gram-positive" antibiotic excluded by the intact outer membrane (OM) permeability barrier, as well as to retapamulin, the surrogate of lefamulin, an antibiotic under development against Gram-positive bacteria. Polymyxin B was used as a comparator. The combination of NAB739 and rifampin was synergistic against ten out of eleven PMR strains of Escherichia coli (Fractional Synergy Indices, FICs, 0.14-0.19) and that of NAB739 and retapamulin against all the tested eleven strains (FICs 0.19-0.25). Against PMR Klebsiella pneumoniae $(n=7)$, the FICs were $0.13-0.27$ for NAB739 + rifampin and 0.14-0.28 for NAB739+ retapamulin. Against Acinetobacter baumannii $(n=2)$, the combination of NAB739 and rifampin had the FIC of 0.09-0.19. Furthermore, NAB739 and meropenem were synergistic (FICs 0.25-0.50) against four out of five PMR strains that were simultaneously resistant to meropenem.
\end{abstract}

\section{Introduction}

Carbapenems have become last-line drugs against extensively drugresistant (XDR) strains of Enterobacteriaceae and Acinetobacter baumannii. Now, the global dissemination of strains carrying carbapenemases has resulted in limited therapeutic options [1-6]. Low serum and urine levels shadow the use tigecycline [5], whereas ceftazidime-avibactam (CZA) is confronted by emerging resistance [6] and is not effective against enzymes that belong to the metallo- $\beta$-lactamase group. Previously in 2010, the World Health Organization (WHO) stated that antibiotic resistance is one of the three greatest threats to human health. Today, and quite alarmingly, only a few novel antibiotics against the XDR strains are under development, as also reported recently by WHO [7]. It follows that, as the search for novel antibacterial agents is more pertinent than ever, it is important to maximize the potential of the current antibiotics.

Polymyxins (polymyxin B [PMB] and colistin, Table 1) are cyclic lipodecapeptide antibiotics, highly basic due to five free amino groups and effective against Gram-negative bacteria such as the majority of Enterobacteriaceae, as well as A. baumannii and Pseudomonas aeruginosa. The discovery of polymyxins was published independently by three laboratories in 1947. Their clinical use was abandoned in the 1960s due to nephrotoxicity and the introduction of better-tolerated drugs belonging to other antibiotic classes. Now, polymyxins have seen a resurgence as the last-line drugs against XDR strains. However, even this new role is in jeopardy. The recent appearance of mobile colistin resistance $(\mathrm{mcr})$ genes has elicited much concern and publicity, but strains resistant to polymyxins by other mechanisms such as those with altered $m g r B$ are also worrisome and should not be overlooked [8-11].

In 2013, Tascini et al. showed that there is notable synergism with colistin and rifampin against polymyxin-resistant (PMR), carbapenemase-producing Klebsiella pneumoniae [12]. Very recently (2018), two publications have highlighted the potential of polymyxin-based combinations against PMR strains in a detailed way. MacNair et al. showed that colistin is notably synergistic in vitro with rifampin, the macrolide antibiotic clarithromycin, minocycline, and novobiocin against $\mathrm{mcr}$

\footnotetext{
* Corresponding author at: Northern Antibiotics Ltd., Technopolis, Tekniikantie 14, FI-02150, Espoo, Finland.

E-mail address: martti.vaara@northernantibiotics.com (M. Vaara).
} 
Table 1

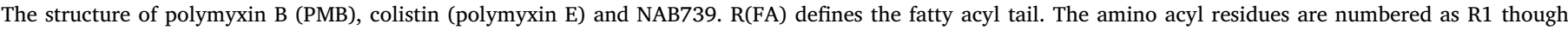
R10. ${ }^{\mathrm{a}, \mathrm{b}}$

\begin{tabular}{|c|c|c|c|c|c|c|c|c|c|c|c|}
\hline Compound & $\mathrm{R}(\mathrm{FA})$ & R1 & R2 & R3 & R4 & R5 & R6 & R7 & R8 & R9 & R10 \\
\hline Polymyxin B (PMB) & Methyloctanoy//methy|heptanoyl & $-\mathrm{Dab}^{+}$ & $-\mathrm{Thr}$ & $-\mathrm{Dab}^{+}$ & $-c y[D a b$ & $-\mathrm{Dab}^{+}$ & -DPhe & -Leu & $-\mathrm{Dab}^{+}$ & $-\mathrm{Dab}^{+}$ & -Thr] \\
\hline Colistin (polymyxin E) & Methyloctanoy $/$ methy|heptanoyl & $-\mathrm{Dab}^{+}$ & - Thr & $-\mathrm{Dab}^{+}$ & $-c y[D a b$ & $-\mathrm{Dab}^{+}$ & -DLeu & -Leu & $-\mathrm{Dab}^{+}$ & $-\mathrm{Dab}^{+}$ & $-\mathrm{Thr}]$ \\
\hline NAB739 & Octanoyl & - & - Thr & -DSer & -cy[Dab & $-\mathrm{Dab}^{+}$ & -DPhe & -Leu & $-\mathrm{Dab}^{+}$ & $-\mathrm{Dab}^{+}$ & -Thr] \\
\hline
\end{tabular}

${ }^{\mathrm{a} A m i n o}$ acyl residues that differ from those in polymyxin B are boxed.

b Abbreviations: cy, cyclic portion indicated with brackets.

strains [13]. Brennan-Krohn et al. described the synergism of colistin with rifampin, the macrolide antibiotic azithromycin, minocycline, fusidic acid, linezolid, and several other drugs against $\mathrm{mcr}$ and other PMR strains [14]. A plausible explanation for the synergism is that even though the PMR strains can grow at higher concentrations of polymyxins than the polymyxin-susceptible wild-type strains, subinhibitory concentrations of polymyxins are still able to damage the outermost permeability barrier, the outer membrane (OM) of the PMR bacteria. Consequently, the damaged $\mathrm{OM}$ allows the entry of drugs normally excluded or partially excluded by the intact enterobacterial OM [13,14]

NAB739 is a novel polymyxin derivative $[15,16]$. It carries only three positive groups, all in strategic positions, instead of five in PMB and colistin (Table 1). Since complicated urinary tract infections (cUTI) caused by XDR strains and proceeding to septic shock are a major concern, compounds like NAB739 may be clinically useful. In a 7-day cynomolgus monkey study, NAB739 yielded at equal doses urinary concentrations ten-fold compared to those of PMB [16]. In a murine E.coli pyelonephritis model, NAB739 was effective at a dose one tenth of that of PMB, even though the MIC of NAB739 against the challenge strain was higher than that of PMB ( $2 \mathrm{mg} / \mathrm{L}$ vs. $0.5 \mathrm{mg} / \mathrm{L})$ [17]. Furthermore, NAB739 is less nephrotoxic to cynomolgus monkeys than PMB [16].

Herein we show that NAB739 acts against PMR strains synergistically with rifampin, the classic compound used in polymyxin synergism studies [15,16,18-20]. Additionally, we report synergism with retapamulin, a pleuromutilin antibiotic and surrogate of lefamulin [21], an antibiotic effective against Gram-positive bacteria only and currently in late clinical development by Nabriva Therapeutics for both oral and intravenous administration [22]. We chose rifampin and retapamulin to represent model antibiotics for the potential synergism, even though many other existing drugs as well as drugs under development may have related synergistic properties with NAB739. Furthermore, we show that NAB739 and a widely-used carbapenem antibiotic, meropenem, are synergistic against PMR strains that are simultaneously resistant to carbapenems. Throughout the studies, PMB was used as a comparator.

\section{Materials and methods}

\subsection{Peptides}

NAB739 sulfate was custom-made by Bachem AG (Bubendorf, Switzerland). The peptide purity, as estimated by HPLC, was $97.3 \%$. Polymyxin B sulfate was from Sigma-Aldrich (St. Louis, MO, USA; product number P0972).

\subsection{Partner antibiotics}

Rifampin, retapamulin and meropenem trihydrate were from Sigma-Aldrich.

\subsection{Bacterial strains}

The polymyxin-resistant strains studied are listed in Table 2. The EN strains are from the ENABLE program, funded by EU and European
Federation of Pharmaceutical Industry Associations (EFPIA). The other polymyxin-resistant strains are from the University of Cardiff collection and originate from Thailand (strains \#1 through \#79) and from Spain (strain 13115). E. coli ATCC 25922, K. pneumoniae ATCC 13883 and A. baumannii ATCC 19606 were used as control strains.

\subsection{Synergism studies}

The synergism was studied by the checkerboard method, where Fractional Inhibitory Concentrations (FICs) $\leq 0.5$ indicate synergism. It has been shown that broth microdilution (BMD) and agar dilution (AD) yield very similar results in polymyxin susceptibility studies [23-27]. We chose AD (according to CLSI [28]) since very recent studies have shown that colistin is heavily bound to plastic in the microwells, especially at low concentrations [29], and that AD is superior to broth dilution methods in colistin susceptibility studies in terms of reproducibility [30]. Also, a recent study on an outbreak of colistin-resistant $K$. pneumoniae relied on the $\mathrm{AD}$ method [31].

The checkerboard agar plates (Mueller-Hinton agar [Becton Dickinson, Oxford, UK]) contained increasing concentrations of NAB739 or PMB $(0.06,0125,0.25,0.5,1,2,4,8,16 \mathrm{mg} / \mathrm{L})$ and the partner antibiotic $(0.25,0.5,1,2,4,8,16 \mathrm{mg} / \mathrm{L})$. No higher concentrations were included since they are irrelevant in clinical settings. The plates also included control plates with no partner antibiotics and those with no NAB739 or PMB (collectively, polymyxins).

\section{Results}

Tables 2 and 3 summarize the synergism studies where rifampin and retapamulin (i.e. the surrogate of lefamulin, see Introduction) were used as partner antibiotics for NAB739 and its comparator, PMB. Table 2 shows the MICs of NAB739, PMB, rifampin, and retapamulin alone as well as the MICs of NAB739 and PMB in the presence of a low concentration of rifampin or retapamulin $(1 \mathrm{mg} / \mathrm{L}$ and $2 \mathrm{mg} / \mathrm{L}$, respectively). The combinations showed significantly reduced MICs for the polymyxin-resistant strains. The exceptions were $E$. coli EN303 that was not susceptible to the combination of polymyxins and rifampin and $A$. baumannii EN284 that was not susceptible to the combination of polymyxins and retapamulin. However, the lack of synergism was probably not due to the lack of the OM-permeability increasing action of polymyxins, because $E$. coli EN303 was susceptible to the combination of polymyxins and retapamulin and A. baumannii EN284 was susceptible to the combination of polymyxins and rifampin.

The MICs both in the absence and in the presence of the partner antibiotic were lower for PMB than for NAB739. In most cases, the difference was 4-fold. On the other hand, the FICs were very similar for NAB739 and PMB (Table 3). The medians and ranges (in parentheses) of the FICs against $E$. coli and $K$. pneumoniae for each combination were: NAB739 + rifampin, 0.19 (0.13-0.27); PMB + rifampin, 0.16 (0.080.25); NAB739 + retapamulin, 0.19 (0.14-0.28); PMB + retapamulin, $0.19(0.09-0.31)$. The synergism of polymyxins with rifampin against EN303 was omitted in the range because this strain apparently had high intrinsic resistance to rifampin. However, EN303 was very susceptible to the synergistic action of polymyxins and retapamulin. Against $A$. baumannii EN284 and EN287, the FICs of NAB + rifampin were 0.19 
Table 2

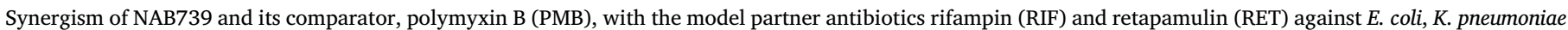
and $A$. baumannii strains that are resistant to polymyxin B (MIC, $>2 \mathrm{mg} / \mathrm{L}$ ). ${ }^{\mathrm{a}}$

\begin{tabular}{|c|c|c|c|c|c|c|c|c|c|c|}
\hline \multirow[b]{2}{*}{ Strain $^{\mathrm{b}}$} & \multirow[b]{2}{*}{ Species } & \multirow[b]{2}{*}{ Resistance type } & \multicolumn{8}{|c|}{ MIC (mg/L) of } \\
\hline & & & NAB739 & PMB & RIF & RET & \multicolumn{2}{|c|}{ in the presence of RIF $(1 \mathrm{mg} / \mathrm{L})$} & \multicolumn{2}{|c|}{ in the presence of RET $(2 \mathrm{mg} / \mathrm{L})$} \\
\hline ATCC 25922 & E. coli & & 1 & 0.5 & 16 & 16 & 2 & 0.5 & 0.5 & 0.25 \\
\hline EN303 & E. coli & Col- $\mathrm{R}^{\mathrm{b}}$ & 16 & 8 & $>16$ & 16 & 8 & 8 & 1 & 0.5 \\
\hline EN481 & E. coli & $m c r-1$ & $>16$ & 8 & $>16$ & 16 & 4 & 0.5 & 4 & 1 \\
\hline EN482 & E. coli & $m c r-1$ & 16 & 8 & 16 & 16 & 4 & 1 & 2 & 1 \\
\hline EN483 & E. coli & $m c r-1$ & $>16$ & 8 & 16 & 8 & 4 & 1 & 2 & 1 \\
\hline EN484 & E. coli & $m c r-1$ & $>16$ & 8 & 8 & 8 & 4 & 0.5 & 2 & 1 \\
\hline EN485 & E. coli & $m c r-1$ & $>16$ & 8 & 16 & 16 & 4 & 1 & 4 & 1 \\
\hline EN486 & E. coli & $m c r-1$ & $>16$ & 8 & 8 & 8 & 2 & 0.5 & 1 & 0.5 \\
\hline$\# 1$ & E. coli & $m c r-1$ & $>16$ & 16 & 16 & $>16$ & 4 & 1 & 4 & 1 \\
\hline$\# 2$ & E. coli & $m c r-1$ & $>16$ & 8 & 16 & $>16$ & 4 & 2 & 2 & 1 \\
\hline$\# 3$ & E. coli & $m c r-1$ & $>16$ & 8 & 16 & 16 & 4 & 2 & 4 & 1 \\
\hline$\# 4$ & E. coli & $m c r-1$ & $>16$ & 16 & 16 & 16 & 4 & 1 & 4 & 1 \\
\hline ATCC 13883 & K. pneumoniae & & 1 & 0.25 & $>16$ & $>16$ & 1 & 0.25 & 0.5 & 0.25 \\
\hline EN245 & K. pneumoniae & Col-R & $>16$ & 16 & $>16$ & $>16$ & 4 & 1 & 4 & 1 \\
\hline EN233 & K. pneumoniae & Col-R & 16 & 16 & 16 & $>16$ & 4 & 1 & 4 & 1 \\
\hline \#38 & K. pneumoniae & $m c r-1$ & $>16$ & $>16$ & $>16$ & $>16$ & 4 & 1 & 4 & 1 \\
\hline \#72 & K. pneumoniae & $m c r-1$ & $>16$ & 16 & $>16$ & $>16$ & 4 & 1 & 4 & 1 \\
\hline \#77 & $K$. pneumoniae & $m c r-1$ & 16 & 8 & $>16$ & $>16$ & 4 & 1 & 4 & 1 \\
\hline \#79 & K. pneumoniae & $m c r-1$ & 16 & 16 & $>16$ & $>16$ & 4 & 1 & 4 & 1 \\
\hline $13 \mathrm{I} 15$ & K. pneumoniae & Col-R & $>16$ & 16 & $>16$ & $>16$ & 1 & 1 & 4 & 1 \\
\hline ATCC 19606 & A. baumannii & & 4 & 0.5 & 4 & $>16$ & 0.125 & 0.125 & 0.5 & 0.25 \\
\hline EN284 & A. baumannii & Col-R & 16 & 16 & 4 & $>16$ & 1 & 0.5 & 16 & 16 \\
\hline EN287 & A. baumannii & Col-R & 8 & 4 & 8 & $>16$ & 0.25 & 0.25 & 4 & 2 \\
\hline
\end{tabular}

a ATCC laboratory control strains are included.

b COL-R, colistin-resistant, undefined mechanism of polymyxin resistance.

and 0.09, respectively and those of $\mathrm{PMB}+$ rifampin 0.13 and 0.19 , respectively.

No synergism between polymyxins and the partner antibiotics was seen against the control strains E. coli ATCC 25922 and $K$. pneumoniae ATCC 13883 (Table 3). However, against A. baumannii ATCC 19606, synergism with FIC values ranging from 0.13 to 0.5 was found. We have previously shown that NAB739 is synergistic with several antibiotics against polymyxin-susceptible A. baumannii strains [32-34].
We also tested the potential synergy of polymyxins with meropenem. No synergism was found against any meropenem-susceptible strains. Against meropenem-resistant strains (Table 4), the FICs of NAB739 and PMB against $K$. pneumoniae EN245 were 0.5 and 0.31 , respectively. Against K. pneumoniae EN233, the corresponding FICs were 0.38 and 0.5 . No synergism was found against $K$. pneumoniae strain 13115. Against A. baumannii EN284, the FICs of NAB739 and PMB were 0.25 and 0.38 , respectively. Against $A$. baumannii EN287, the

Table 3

Synergism of NAB739 and its comparator (PMB) with two model partners (rifampin, retapamulin) against polymyxin-resistant strains, as shown by using FIC indices. ${ }^{\mathrm{a}}$

\begin{tabular}{|c|c|c|c|c|c|c|}
\hline \multirow[t]{2}{*}{ Species } & \multirow[t]{2}{*}{ Isolate } & \multirow[t]{2}{*}{ Property } & \multicolumn{4}{|c|}{ FIC of a combination of polymyxins with two model partners (RIF and RET) } \\
\hline & & & NAB739+RIF & $\mathrm{PMB}+\mathrm{RIF}$ & NAB739+RET & $\mathrm{PMB}+\mathrm{RET}$ \\
\hline \multirow[t]{12}{*}{ E. coli } & ATCC 25922 & WT & 0.75 & 0.75 & 0.56 & 0.52 \\
\hline & EN303 & Col-R & 0.50 & 0.50 & 0.19 & 0.19 \\
\hline & EN481 & $m c r-1$ & 0.19 & 0.08 & 0.19 & 0.19 \\
\hline & EN482 & $m c r-1$ & 0.19 & 0.16 & 0.19 & 0.19 \\
\hline & EN483 & $m c r-1$ & 0.14 & 0.16 & 0.25 & 0.25 \\
\hline & EN484 & $m c r-1$ & 0.16 & 0.16 & 0.25 & 0.31 \\
\hline & EN485 & $m c r-1$ & 0.19 & 0.19 & 0.19 & 0.19 \\
\hline & EN486 & $m c r-1$ & 0.16 & 0.16 & 0.19 & 0.25 \\
\hline & 1 & $m c r-1$ & 0.19 & 0.19 & 0.25 & 0.13 \\
\hline & 2 & $m c r-1$ & 0.19 & 0.25 & 0.25 & 0.25 \\
\hline & 3 & $m c r-1$ & 0.19 & 0.25 & 0.25 & 0.25 \\
\hline & 4 & $m c r-1$ & 0.19 & 0.19 & 0.25 & 0.19 \\
\hline \multirow[t]{8}{*}{ K. pneumoniae } & ATCC 13883 & WT & 0.56 & 0.52 & 0.56 & 0.63 \\
\hline & EN245 & Col-R & 0.13 & 0.08 & 0.14 & 0.09 \\
\hline & EN233 & Col-R & 0.27 & 0.08 & 0.26 & 0.09 \\
\hline & 38 & $m c r-1$ & 0.13 & 0.07 & 0.19 & 0.09 \\
\hline & 72 & $m c r-1$ & 0.13 & 0.08 & 0.16 & 0.09 \\
\hline & 77 & $m c r-1$ & 0.26 & 0.08 & 0.26 & 0.19 \\
\hline & 79 & $m c r-1$ & 0.26 & 0.08 & 0.28 & 0.13 \\
\hline & 13115 & Col-R & 0.13 & 0.07 & 0.14 & 0.09 \\
\hline \multirow[t]{3}{*}{ A. baumannii } & ATCC 19606 & WT & 0.13 & 0.31 & 0.19 & 0.50 \\
\hline & EN284 & Col-R & 0.19 & 0.13 & 1 & 0.75 \\
\hline & EN287 & Col-R & 0.09 & 0.19 & 0.5 & 0.56 \\
\hline
\end{tabular}

${ }^{\text {a }}$ FIC values $\leq 0.5$ indicate synergism and are shown in cursif. 
Table 4

Synergism of NAB739 and its comparator, PMB, with meropenem against polymyxin-resistant strains that are simultaneously carbapenem-resistant. ${ }^{\mathrm{a}}$

MIC (mg/L) of MIC of meropenem at the NAB739 concentration (mg/L) of FIC MIC of meropenem at the PMB concentration (mg/L) of

\begin{tabular}{|c|c|c|c|c|c|c|c|c|c|c|c|c|}
\hline Species & Strain & meropenem & 1 & 2 & 4 & 8 & & 1 & 2 & 4 & 8 & \\
\hline K. pneumoniae & EN245 & 4 & 4 & 4 & 2 & 1 & 0.50 & 4 & 4 & 1 & 0.25 & 0.31 \\
\hline K. pneumoniae & EN233 & 8 & 8 & 8 & 2 & 1 & 0.38 & 8 & 4 & 2 & 1 & 0.50 \\
\hline K. pneumoniae & 13115 & 8 & 8 & 8 & 8 & 8 & 0.75 & 8 & 8 & 8 & 8 & 0.75 \\
\hline A. baumannii & EN284 & $>16$ & $>16$ & 8 & 4 & 4 & 0.25 & 16 & 16 & 9 & 9 & 0.38 \\
\hline A. baumannii & EN287 & $>16$ & 4 & 0.5 & 0.25 & $\mathrm{NA}^{\mathrm{b}}$ & 0.25 & 0.25 & 0.25 & NA & NA & 0.27 \\
\hline
\end{tabular}

${ }^{\text {a }}$ FIC values $\leq 0.5$ indicate synergism and are illustrated in cursif.

b Not applicable, the peptide itself inhibited the growth.

corresponding MICs were 0.25 and 0.27 . These findings merit more extensive studies on the potential of the combination of polymyxins with meropenem and other carbapenems against strains that are simultaneously resistant to polymyxins and meropenem.

\section{Discussion}

It has long been known that polymyxins increase the permeability of the $\mathrm{OM}$ and permeabilize it to other drugs. This property is most easily recognizable with derivatives such as polymyxin $B$ nonapeptide (PMBN), NAB7061 and NAB741 that lack any direct antibacterial activity $[15,16,18-20,32,35]$, Several other polycationic peptides, such as linear lipopeptide paenipeptins and unacylated tridecaptin share this property of polymyxins and drastically sensitize the target bacteria to rifampin [36,37], clarithromycin [36], and many other antibiotics [37]. Besides the traditional antibiotics, certain novel antibiotics under development, such as the lantibiotic NAI-107, are synergistic with polymyxins [38].

It is quite noteworthy that the OM-permeabilizing activity of polymyxins, and perhaps that of several other polycationic peptides, extend to enterobacterial strains that display either mutational or acquired resistance to polymyxins (PMR) strains [12-14]. Here, we showed that NAB739, too, belongs to the group of agents that permeabilize the OM of PMR strains to other drugs. Even though PMR strains are still comparatively rare [39], they pose a potential threat. NAB739 has advantages over PMB, such as better efficacy (as shown in murine urinary tract infection; efficacious dose, one tenth of that of PMB) as well as better tolerability (as studied in cynomolgus monkeys).

Approx. 6.3 million patients were treated in 2013 in the hospital wards in Europe, the USA and Japan due to cUTIs caused by E. coli and $K$. pneumoniae [40]. These two bacteria cause $80 \%$ of all cUTIs treated in hospitals. In the future, many more of those infections will be caused by the XDR strains than is already reported [40]. NAB739 may prove to be a reliable "next generation" polymyxin for clinical use and find much more role over polymyxins in the therapy of infections caused by XDR strains. Whether it is more effective and better tolerated than PMB in the treatment of cUTIs and resulting bacteraemias and septic infections (as the preclinical studies suggest), remains to be investigated.

\section{Declaration of interest} Ltd.

TV and MV are employees and shareholders of Northern Antibiotics

\section{References}

[1] J.A. Otter, P. Burgess, F. Davies, S. Mookerjee, et al., Counting the cost of an outbreak of carbapenemase-producing Enterobacteriaceae; an economic evaluation from a hospital perspective, Clin. Microbiol. Infect. 23 (2017) 188-196.

[2] M. Tumbarello, A. Rafaella Losito, H. Giamarellou, Optimizing therapy of carbapenem-resistant Enterobacteriaceae infections, Curr. Opin. Infect. Dis. 31 (2018), https://doi.org/10.1097/QCO.0000000000000493.

[3] R.A. Bonomo, E.M. Burd, J. Conly, B.M. Limbago, et al., Carbapenem-producing organisms: a global scource, Clin. Infect. Dis. 66 (2018) 1290-1297.
[4] M. Bassetti, D.R. Giagobbe, G. Giamarrellou, C. Viscoli, et al., Management of KPCproducing Klebsiella pneumoniae infections, Clin. Microb. Infect 24 (2018) 133-144.

[5] T.L. Doan, H.B. Fung, D. Mehta, P.F. Riska, Tigecycline: a glycylcycline antimicrobial agent, Clin. Ther. 28 (2006) 1079-1106.

[6] R.K. Shields, L. Chen, S. Cheng, K.D. Chavda, et al., Emergence of ceftazidimeavibactam resistance due to plasmid-borne $b{ }_{b} a_{\mathrm{kpc}-3}$ mutations during treatment of carbapenem-resistant Klebsiella pneumoniae infections, Antimicrob. Agents Chemother. 61 (2017) e02097-16.

[7] https://www.who.int/medicines/areas/rational_use/antibacterial_agents_clinical_ development/en/ (Accessed on 30 October 2018).

[8] Y.Y. Liu, Y. Wang, T.R. Walsh, L.X. Yi, et al., Emergence of plasmid-mediated colistin resistance mechanism MCR-1 in animals and human beings in China: a microbiological and molecular biological study, Lancet Infect. Dis. 16 (2016) 161-168.

[9] S. Baron, L. Hadjadj, J.M. Rolain, A.O. Olaitan, Molecular mechanisms of polymyxin resistance: knowns and unknowns, Int. J. Antimicrob. Agents 48 (2016) 583-591.

[10] K. Jeannot, A. Bolard, P. Plésiat, Resistance to polymyxins in Gram-negative organisms, Int. J. Antimicrob. Ag. 49 (2017) 526-535.

[11] L. Poirel, A. Jayol, P. Nordmann, Polymyxins: antibacterial activity, susceptibility testing, and resistance mechanisms encoded by plasmids or chromosomes, Clin. Microbiol. Rev. 30 (2017) 557-596.

[12] C. Tascini, F. Tagliaferri, T. Giani, A. Leonildi, S. Flammini, et al., Synergistic activity of colistin plus rifampin against colistin-resistant KPC-producing Klebsiella pneumoniae, Antimcrob. Agents Chemother 57 (2013) 3990-3993.

[13] C.R. MacNair, J.M. Stokes, L.A. Carfrae, et al., Overcoming mcr-1 mediated colistin resistance with colistin in combination with other antibiotics, Nat. Commun. 9 (2018) 458-465, https://doi.org/10.1038/s41467-018-02875-z.

[14] T. Brennan-Krohn, A. Pironti, J.E. Kirby, Synergistic activity of colistin-containing combinations gainst colistin-resistant Enterobacteriaceae, Antimicrob. Agents Chemother. 62 (2018) e00873-18.

[15] M. Vaara, Novel derivatives of polymyxins, J. Antimicrob. Chemother. 68 (2013) 1213-1219.

[16] M. Vaara, New polymyxin derivatives that display improved efficacy in animal infection models as compared to polymyxin B and colistin, Med. Res. Rev. 38 (2018) 1661-1673, https://doi.org/10.1002/med.21494.1-13 2018.

[17] M. Vaara, T. Vaara, C. Vingsbo Lundberg, The polymyxin derivatives NAB739 and NAB815 are more effective than polymyxin B in murine Escherichia coli pyelonephritis, J. Antibicrob. Chemother 73 (2018) 452-455.

[18] M. Vaara, T. Vaara, Sensitization of Gram-negative bacteria to antibiotics and complement by a nontoxic oligopeptide, Nature 303 (1983) 526-528.

[19] M. Vaara, T. Vaara, Polycations sensitize enteric bacteria to antibiotics, Antimicrob. Agents Chemother. 24 (1983) 107-113.

[20] M. Vaara, Agents that increase the permeability of the outer membrane, Microbiol. Rev. 56 (1992) 395-411.

[21] M.P. Veve, J.L. Wagner, Lefamulin: review of a promising novel pleuromutilin antibiotic, Pharmacother 38 (2018) 935-946.

[22] https://www.nabriva.com/pipeline-research (Accessed on 30 October 2018).

[23] J.R. Lo-Ten-Foe, A.M. de Smet, B.M.W. Diederen, J.A.J. Kluytmans, P.H.J. van Keulen, Comparative evaluation of the VITEK 2, disk diffusion, Etest, broth microdilution, and agar dilution susceptibility testing methods for colistin in clinical isolates, including heteroresistant Enterobacter cloacae and Acinetobacter baumannii strains, Antimicrob. Agents Chemother. 51 (2007) 3726-3730.

[24] A.C. Gales, R.N. Jones, H.S. Sader, Contemporary activity of colistin and polymyxin B against a worldwide collection of Gram-negative pathogens: results from the SENTRY Antimicrobial Surveillance Program (2006-09), J. Antimicrob. Chemother. 66 (66) (2011) 2070-2074.

[25] J.A. Hindler, R.M. Humphries, Colistin MIC variability by method for contemporary clinical isolates of multidrug-resistant Gram-Negative bacilli, J. Clin. Microbiol. 51 (2013) 1678-1684.

[26] K. Dafopoulou, O. Zarkotou, E. Dimitroulia, C. Hadjichristodoulou, et al., Comparative evaluation of colistin susceptibility testing methods among carbapenem-nonsusceptible Klebsiella pneumoniae and Acinetobacter baumannii clinical isolates, Antimicrob. Agents Chemother. 59 (2015) 4625-4630.

[27] J. Lellouche, D. Schwartz, N. Elmalech, M. Amar Ben Dalak, et al., Combining VITEK 2 with colistin agar dilution screening assists timely reporting of colistin susceptibility, Clin. Microbiol. Infect. (2018), https://doi.org/10.1016/j.cmi.2018. 09.014 . 
[28] CLSI, Methods for Dilution Antimicrobial Susceptibility Tests for Bacteria That Grow Aerobically, approved standard-tenth edition, (2015), pp. M07-A10.

[29] M. Karvanen, C. Malmberg, P. Lagerbäck, L.E. Friberg, O. Cars, Colistin is extensively lost during standard in vitro experimental conditions, Antimicrob. Agents Chemother. 61 (2017) 61, https://doi.org/10.1128/AAC.00857-179.

[30] A. Turlej-Rogacka, B.B. Xavier, L. Janssens, C. Lammens, et al., Evaluation of colistin stability in agar and comparison of four methods for MIC testing of colistin, Eur. J. Clin. Microbiol. Infect. Dis. 37 (2018) 345-353.

[31] J.A. Otter, M. Doumith, F. Davies, S. Mookerjee, et al., Emergence and clonal spread of colistin resistance due to multiple mutational mechanims in carbapenemaseproducing Klebsiella pneumoniae in London, Sci. Rep. 7 (2017) 12711, https://doi. org/10.1038/s41598-017-12637-4.

[32] M. Vaara, J. Fox, G. Loidl, O. Siikanen, J. Apajalahti, et al., Novel polymyxin derivatives carrying three positive charges are effective antibacterial agents, Antimicrob. Agents Chemother. 52 (2008) 3229-3236.

[33] M. Vaara, T. Vaara, Structure-activity studies on novel polymyxin derivatives that carry only three positive charges, Peptides 31 (2010) 2318-2321.

[34] M. Vaara, T. Vaara, J.M. Tyrrell, Structure-activity studies on polymyxin derivatives carrying three positive charges only reveal a new class of compounds with strong antibacterial activity, Peptides 91 (2017) 8-12.

[35] M. Vaara, O. Siikanen, J. Apajalahti, J. Fox, et al., A novel polymyxin derivative that lacks the fatty acid tail and carries only three positive charges has strong synergism with agents excluded by the intact outer membrane, Antimicrob. Agents Chemother. 54 (2010) 3341-3346.

[36] S.H. Moon, X. Zhang, G. Zheng, D.G. Meeker, M.S. Smeltzer, E. Huang, Novel linear lipopeptide paenipeptins with potential for eradicating biofilms and sensitizing Gram-Negative bacteria to rifampicin and clarithromycin, J. Med. Chem. 60 (2017) 9630-9640.

[37] S.A. Cochrane, J.C. Vederas, Unacylated tridecaptin $A_{1}$ acts an effective sensitizer of Gram-negative bacteria to other antibiotics, Int. J. Antimicrob. Agents 44 (2014) 493-499.

[38] C. Brunati, T.T. Thomsen, E. Gaspari, S. Maffioli, et al., Expanding the potential of NAI-107 for treating serious ESKAPE pathogens: synergistic combinations against Gram-negatives and bactericidal activity against non-dividing cells, J. Antimicrob. Chemother. 73 (2018) 414-424.

[39] https://sentry-mvp.jmilabs.com/ (accessed on Sep 15, 2018).

[40] T.M. Stewart, K. Dorfman, Pharmacol. Inf. Dis. Dec. Resourc. (2015) 1-209. 